\title{
Study on Selective Leakage Protection System of Mine Low- voltage Grid Based on Internet of Things
}

\author{
Liu Zhongfu, Han Guiying and Shi Lixin \\ College of Information \& Communication Engineering \\ Dalian Nationalities University, Liaoning Dalian, China \\ lzhongfu@163.com
}

\begin{abstract}
Leakage is one of the most common fault forms of mine low-voltage grid protection. With the continuous increase of automation degree of mine, the leakage protection system of mine low-voltage grid needs to be monitored and managed comprehensively; as a response to this demand, the selective leakage protection system of mine low-voltage grid is explored and studied in this paper from the perspective of practical application based on Internet of Things. In this paper, the design of leakage protection system of mine lowvoltage grid is proposed based on the combination of ZigBee protocol the framework of Internet of Things, and the perception layer of Internet of Things is constructed by ZigBee technology to collect and transmit the related data. Meanwhile, the low-voltage leakage fault principle of the mine and the variation law of zero-sequence current and zerosequence voltage mine are analyzed in this paper; on this basis, a selective leakage protection scheme based on the additional DC power detection and zero-sequence power direction is proposed, in which additional DC power principle is applied to the main feeder switch, the protection principle of zero-sequence power direction is applied to the branch feeder switch, and the branch leakage is interpreted by Fourier algorithm. Through experimental verification, the performance indicators of leakage protection system are consistent with standard requirements, with the small error, which can meet the requirements of the mine grid leakage protection. Internet of Things can realize ZigBee networking and transmit the leakage information of the grid the host computer through ZigBee network.
\end{abstract}

Keywords: Internet of Things, mine low-voltage grid, selective leakage protection, ZigBee

\section{Introduction}

$6 \mathrm{KV}$ high-voltage power of the mine is led from the ground substation by bus to the underground substation. The voltage is lowered down to low voltage, $1140 \mathrm{~V}$ and $660 \mathrm{~V}$, through transformer of each substation, and then supplied to the low-voltage electrical equipment in mining area. The low-voltage power supply system of underground mine consists of multiple small power-supply units that are relatively independent. A small power-supply unit is composed of transformer, low-voltage feeder switch, magnetic starter, mining cables, motors and other components. Figure 1 shows a complete unit model of electrical power supply.

In the figure, $\mathrm{T}$ is the power transformer, DW is low-voltage feeder switch and QC is the magnetic starter.

With continuous expansion of mine capacity, the number of small power-supply units of mine low-voltage grid is increased. The normal operation of the power supply unit is related to the system stability and safe operation of the entire mine low-voltage grid. Leakage is one of the major faults of small low-voltage power supply unit, accounting for about $70 \%$ of the fault, which not only results in personal electric shock, but also leads to 
single-phase ground fault, thus developed into interphase short circuit, and the resulted arc may cause gas and coal explosion. To ensure personal safety, and reduce explosion danger of gas and coal caused by leakage, selective leakage protection device must be installed in a small power-supply unit.

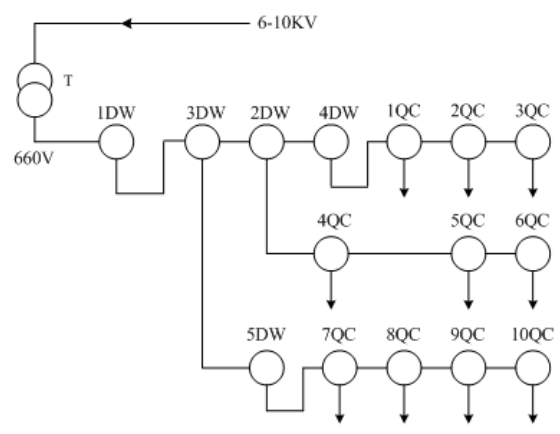

\section{Figure 1. The Electrical Model of Underground Low-Voltage Power Supply Unit}

After a leakage fault of mine low-voltage grid occurs, the response and treatment for the fault will take some time. In order to eliminate the leakage fault effectively, and ensure the stability of power supply to maximum extent, selective leakage protection technology based on Internet of Things is introduced into the control system of mine lowvoltage grid.

The selective leakage protection system of mine low-voltage grid can protect the grid automatically according to the operation of the power; meanwhile, real-time operation data of small power-supply units are obtained quickly and accurately by an effective means of communication, and the control command of control center is accurately transmitted to the remote leakage protection system. The introduction of Internet of Things technology to low-voltage protection system of grid can further improve the accuracy of communication, thus improving the efficiency of leakage protection system.

This paper analyzes the theory of mine Low leakage failure and zero-sequence current and zero sequence voltage changes, on this basis, we propose a selective leakage protection scheme based on the additional DC power detection and zero-sequence power direction, the program of the total feed the principle use of additional DC power switch at the use of the principle of protection with zero-sequence power direction of feed switch at the branch, the branch leakage Fourier algorithm interpretation. The design of leakage protection system of mine low-voltage grid is proposed based on the combination of ZigBee protocol the framework of Internet of Things, and the perception layer of Internet of Things is constructed by ZigBee technology to collect and transmit the related data.

\section{Application of Internet of Things Technology in the Selective Leakage Protection System of Mine Low-Voltage Grid}

\subsection{Overview of Internet of Things Technology}

Internet of Things is a further expansion of internet and communication network. Advanced perception technologies are used to further identify the real world and the perceived information by the transmissions of various communication networks, and information processing and data exploration are conducted [1]. And then the exchange of information and seamless connection between person and person, person and thing, as well as thing and thing is realized, and real-time monitoring, scientific management and decision-making are also realized. Internet of Things is able to conduct a comprehensive perception of the outside world, which can make the perceived information transmitted 
and analyzed reliably and stably. Internet of Things is the product of the integration of sensor network and communication network, rather than independent network. With the further development of technology, Internet of Things is can be applied to the leakage protection system of mine low-voltage grid [2].

\subsection{Structure of Selective Leakage Protection System of Mine Low-Voltage Grid Based On Internet of Things}

The selective leakage protection system of mine low-voltage grid based on Internet of Things is shown as Figure 2. The structure of the system contains three levels, namely, perception level, network level and application level.

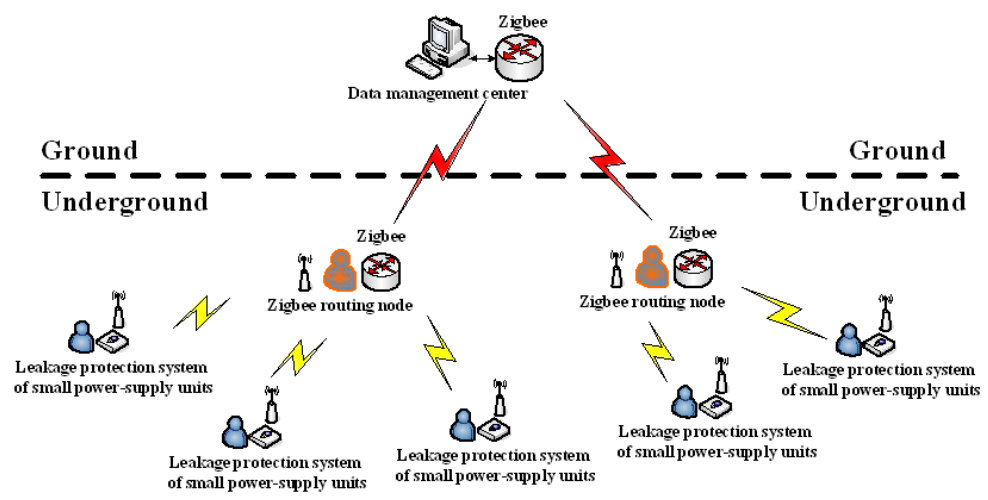

Figure 2. Structure of Selective Leakage Protection System of Mine LowVoltage Grid Based on Internet of Things

Perception level is mainly responsible for perceiving and collecting information. Perception level consists of sensor and short-range network transmission system. Perception level adopts ZigBee network technology, which is composed of an aggregation node (ZigBee network terminal) and several branch nodes (ZigBee collector). ZigBee collector collects the information about the operation and the fault of different power supply units of mine low-voltage power grid, and transmits it to the ZigBee network terminal.

Aggregation node adopts the ZigBee device of full functionality, and CC2430 is selected as module, which acts as coordinator in the system. The following functions are realized:

(1) Network and wireless transceiver are established;

(2) Checksum is calculated.

(3) Reference nodes are positioning nodes of computer data configuration are transmitted.

(4) Positioning node coordinates of the requested configuration from computer data.

Low power, low cost, portability and other factors are taken into account in this system, in which based on Single Chip Microcomputer CC2430 of short-range wireless communication as core, CC2430 core board is designed, and wireless transmission of information is completed between core boards of the network.

The main function of the network level is to realize reliable and efficient transmission of the data from perception level. The network level of this system is a transmission network constituted by ZigBee network [3].

The main function of application level is to conduct further integration, classification and summarization of the data integrated preliminarily in network level, and combines the final data information with the terminal of application platform, to complete the 
comprehensive utilization of data. This part is mainly to monitor the software of host computer [4].

\section{Analysis of Leakage Principle of Low-Voltage Network}

Neutral point of the insulation system is applied in mine low-voltage network. It remains three-phase symmetrical without any change in line voltage no matter which type of leakage fault power occurs. Single-phase and two-phase leakages are asymmetrical faults; after the fault, each relative power of grid is no longer symmetrical, and the neutral point of transformer is displaced, resulting in ground voltage (zero-sequence voltage); if the system has zero-sequence circuit, there is zero-sequence current in the circuit[5].

Single-phase leakage fault accounts for $85 \%$ of the total leakage faults in low-voltage power grid of the mine, and if single-phase leakage fault is not removed in time, it will develop into short-circuit fault, which is more serious. Single-phase leakage is taken as research object to make analysis of the variation law of the fault parameters, as shown in the following.

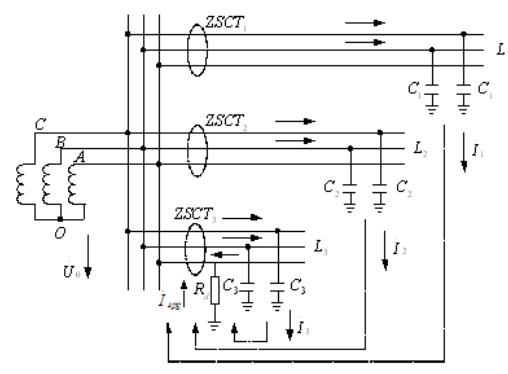

\section{Figure 3. Single-Phase Grounding Capacity Current Distribution In Mine Low-Voltage Grid}

In the insulation power supply system of neutral point, the leakage current distribution characteristics are shown in Figure 4, in which a typical pattern of China's coal mine lowvoltage grid is shown, composed of three branches $L_{1}, L_{2}$ and $L_{3}$, and $Z S C T_{1}$ and $Z S C T_{2}$, $Z \mathrm{DCT}_{3}$ are installed in the zero-sequence current transformer of each branch respectively. Due to the high level of insulation in low-voltage grid, resistance to the ground of each phase is assumed as $\infty$. Assuming the distributed capacitance of each branch is equal, denoted by $C_{1}, C_{2}$ and $C_{3}$ respectively. When ground fault occurs in A phase of the branch $L_{3}\left(R_{d}=0\right)$, all the potentials to ground of $A$ phase in the whole grid are zero. The potential of the neutral point $0 U_{0}=-U_{A}$, and the voltages to the ground of $B$ and phases become line voltages, namely

$$
\begin{aligned}
& U_{B}^{\prime}=U_{0}+U_{B}=\sqrt{3} U_{A} e^{-j 150^{\circ}} \quad(\text { Equation 1) } \\
& U_{C}^{\prime}=U_{0}+U_{C}=\sqrt{3} U_{A} e^{-j 150^{\circ}} \text { (Equation 2) }
\end{aligned}
$$

The grounding capacitor of A phase in each branch is connected by short circuit, but the grounding capacitors of $\mathrm{B}$ and $\mathrm{C}$ phases form capacitance current under the effect of respective ground voltages. Taking branch $L_{1}$ as an example, $I_{1}, I_{2}$ and $I_{3}$ return to the power from the ground to constitute grounding currents, namely the values are

$$
I_{j d}=I_{1}+I_{2}+I_{3}=3 j \omega U C_{\Sigma} \quad(\text { Equation 3) }
$$

when $I_{1}$ and $I_{2}$ pass from the primary side of zero-sequence current transformers $Z S C T_{1}$ and $Z S C T_{2}$ respectively, the current through zero-sequence current transformer $Z S C T_{3}$ is the vector sum of $I_{j d}$ and $I_{3}$, denoted as $I_{f}$, namely 


$$
I_{f}=-I_{j d}+I_{3}=-\left(I_{1}+I_{2}\right)=-3 j \omega U_{0}\left(C_{1}+C_{2}\right) \quad \text { (Equation 4) }
$$

The characteristics of the single-phase grounding capacitance current are:

(1) The current flowing through the zero-sequence current transformer of non-fault branch is the capacitive current of this branch, of which the phase exceeds $U_{0}$ by $90^{\circ}$;

(2) The current flowing through the zero-sequence current transformer of fault branch is the sum of all the capacitance currents of other non-fault branches, of which the phase is lagged $90^{\circ}$ by $U_{0}$ (the zero-sequence current generated by fault branch flows through the zero-sequence transformer twice, and therefore it is self-canceling for transformer);

(3) In general, mine low-voltage grid is composed of multiple branches. Therefore, the capacitance current flowing through fault branch may be higher than that of any branch.

These characteristics are the main theoretical basis for the general selective leakage protection technology [6].

\section{Leakage Protection of Mine Low-Voltage Grid}

Currently, the major leakage protections of mine low-voltage grid mainly include additional DC leakage protection, zero-sequence current leakage protection, zerosequence voltage protection, and zero-sequence power directional protection.

Additional DC leakage protection can cover the entire low-voltage power supply unit, by which the fault trip is not influenced by fault type, time and place of occurrence. However, additional DC leakage protection is not selective; whenever and wherever the leakage occurs in low-voltage power supply unit, it will lead to the trip of main switch, causing widespread power outages. Zero-sequence current leakage protection has selectivity as it selects leakage current based on the fact that zero-sequence current magnitude in fault branch is larger that in non-fault branch. But zero-sequence current protection cannot exclude the interference from the unbalance of current transformer, with low detection sensitivity. Zero-sequence voltage protection makes use of the zerosequence voltage amplitude in leakage to reflect the degree of insulation of the grid to ground, but the action resistance value of this leakage protection is not fixed, susceptible to the interference of the capacitor; it is non-selective, and it will not operate when gridto-ground impedance drops, with a relatively narrow range of application. Zero-sequence power directional protection can not only determine whether asymmetric power leakage occurs, but also determine the leakage branch, with selecting function of leakage [7].

The system adopts the combination of an additional DC power and zero-sequence power direction, in which additional DC power principle is applied to the main feeder switch, the protection principle of zero-sequence power direction is applied to the branch feeder switch to realize selective leakage protection of mine low-voltage grid. The interpreting criterion for additional DC power protection is insulation resistance value; when the insulation resistance is less than the action value, it is determined as leakage, whereas no leakage. Zero-sequence power direction method is to judge the direction according to the calculated power; the power is greater than 0 in fault branch, while the power in non-fault branch is less than 0 .

\subsection{Additional DC Leakage Protection}

China's coal mine grids all adopt neutral-point insulation operation mode of power transformer, and supply power by cables. Because of the special working condition in coal mine and humid environment, higher requirements must be made for the insulation of applied electrical equipment and cables. Nevertheless, there is still a risk of leakage for the operated electrical equipment and power cables. Especially for the surface cables with 
greater probability of being squeezed or smashed, the insulation is more susceptible to damage, with greater possibility of leakage.

From the analysis above, the system must measure or monitor the insulation at the load side of the motor in magnetic starter. When the insulation resistance to ground drops to a predetermined value, the leakage blocking protection device works, to close the corresponding vacuum contactor control circuit, so that it cannot deliver electricity. Leakage blocking only monitors the insulation state of the charging in power-off condition, and when the main circuit works, it will exit from the detection state. As leakage blocking protection makes fault power-supply circuit not be put into the work, it reduces the chance of exposed sparks. From the perspective of leakage fault searching, leakage blocking protection is helpful for interpreting the fault scope and shortening the searching time.

Magnetic starter controls the start and stop of the motor directly, with a short distance of the power supply, of which the leakage fault during work will be protected by superior feeder switch, and leakage fault in power-off state shall be protected by leakage blocking protection unit of magnetic starter. Therefore, from the perspective of security, magnetic starter must be equipped with leakage blocking protection.

Leakage blocking protection unit is composed of DC detection power, DC detection circuit, signal sampling circuit and implementation and control circuit, of which the detection principle is shown in Figure 4.

In the figure, DPS is DC detection power, which comes from the phase voltage of main circuit, and output from the transformer, rectifier, filter and regulator. DC detection circuit is composed of high-voltage silicon stack GD, normally closed contact $\mathrm{KM}$ in main contractor, normally open contact KL of leak detection relay, DC detection power DPS, action resistance adjustment potentiometer WR, grid insulation resistance to ground (RU, $\mathrm{RV}$ and RW) and grid. As the load side of magnetic starter motor is directly connected to motor, and for the current of the DC detection, the three-phase winding of the motor is equivalent to the connection with a three-phase grid, so that insulation condition of each phase of the motor winding in three-phase grid can be detected by the DC detection circuit connected to only one phase of the grid.

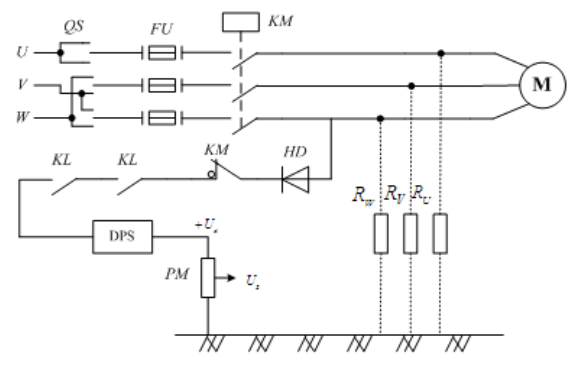

\section{Figure 4. Detection Principle of Additional DC Power Supply}

Before closing circuit, when the system determines that the setting is correct without leakage test or short-circuit test, the normally open contact of leakage detection relay KL is pulled in, DC detection circuit is connected through the normally closed auxiliary contact of contactor $\mathrm{KM}$ to start testing the grid insulation resistance, and with the leakage detecting signal $U_{L}$ is changed with the varying of the insulation resistance value to ground. When the insulation resistance to ground is less than the set value of leakage blocking, CPU sends out the blocking signal, to make the motor unable to start; otherwise, closing starter is allowed. According to Figure 4, the expression of UL can be drawn as

$$
U_{L}=\frac{U_{d} \times R_{P M}}{R_{X} \times \Sigma R_{i}} \quad(\text { Equation } 5)
$$


After the system is determined, $\Sigma R_{i}$ is a fixed value. Therefore, $U_{L}$ increases linearly as $\mathrm{RX}$ declines, when $U_{L}$ is greater than the set value, CPU sends out leakage blocking signal, realizing leakage locking function of the motor. So when the detected voltage is constant, the leakage resistance value of the system is very stable.

High-voltage silicon stack is designed to prevent the counter electromotive force of the motor into the detection circuit. After the trip of main circuit, the rotor of motor continues to rotate to generate an electromotive force due to inertia, and high-voltage silicon stack can prevent this electromotive force fleeing to detection circuit to damage other components.

In control circuit, SCM software is used to block motor starting; when the occurrence of leakage is detected, the software flag bit is 1 and the motor starting circuit is disconnected, so that the motor cannot start, thus completing the leakage blocking protection.

\subsubsection{Determination of Additional DC Voltage and Current}

It is obvious that the prerequisite for the linear growth of $U_{L}$ with the decline of RX is that direct detection voltage $U$ must be a constant value. In other words, the voltage fluctuates in the specified range $75 \% U_{N} \sim 115 \% U_{N}$; $\mathrm{U}$ must be a fixed value in order to ensure the stability of the action resistance.

For different voltage levels of the grid, the withstand voltages of insulation materials are different, of which the true insulation condition can only be reflected by the application of different DC voltages. In the permitted range of withstand voltage of insulation materials, the higher the voltage of the additional DC power supply is, the closer to the actual value the measured resistance value is. Taking $660 \mathrm{~V}$ grid as an example, the test data of the insulation resistance and additional DC voltage are shown in Table 1. The insulation resistance to ground of the grid measured by this method is referred to as DC resistance.

Table 1. VARIATION of DC Insulation Resistance $R$ with the Additional DC Voltage U

\begin{tabular}{cccccccc}
\hline $\mathrm{U}(\mathrm{V})$ & 20 & 36 & 52 & 82 & 120 & 177.5 & 235 \\
\hline $\mathrm{R}(\mathrm{K} \Omega)$ & 100 & 800 & 742.8 & 672.1 & 571 & 522 & 489.5 \\
& 0 & & & & & & \\
\hline
\end{tabular}

According to regulations, DC detection voltage must meet the requirements of the intrinsically safe circuit; otherwise, it will lead to new risks. Leakage blocking protection is to test the insulation before the motor is started. Once leakage or single-phase ground fault is found, the spark generated in the DC detection circuit is not allowed to cause an explosion of gas and coal. Moreover, distributed capacitance exists in power lines to ground, and this capacitance always has full charge under the effect of additional DC detection power supply; in case of single-phase ground, the capacitance shall discharge through grounding point, and the energy of discharging spark should be less than the minimum energy required to cause gas or coal explosion.

In addition, the safety of maintenance workers should be considered. After power outage, workers may carry out maintenance work of electrical equipment; if the DC voltage is too high, it may be a shock hazard when a person contacts it. Therefore, the detection voltage should not be too high. 


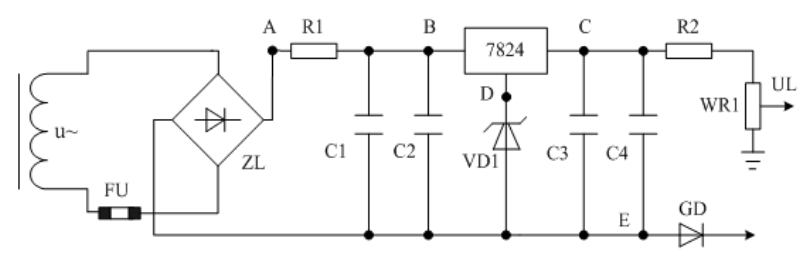

Figure 5. Additional DC Detection Circuit

As can be seen from the above analysis, when the supply voltage of DC detection circuit is set as $50 \mathrm{~V}$, allowed capacitance to ground is $1.5 U_{f}$, which can meet the security requirement for underground low-voltage network. In the design of system, the DC detection voltage is set as $40 \mathrm{~V}$. Besides, direct detection current should not be too large as well; when the power is supplied at the end of the main circuit, single-phase grounding current should be no more than $2 \mathrm{~mA}$, and in the design of the system, $1 \mathrm{~mA}$ is considered, which meets the requirement of intrinsically safe circuit. Hardware circuit is shown in Figure 5.

A three-phase regulator is used to test the voltage value of each point with $75 \% U_{N}, U_{N}$ and $115 \% U_{N}$, as shown in Table 2 .

Table 2. Tested Values of Leakage Signals under Different Voltages

\begin{tabular}{cccccc}
\hline $\begin{array}{l}\mathrm{U} \sim \\
(\mathrm{V})\end{array}$ & $\begin{array}{c}\mathrm{U}_{\mathrm{A}} \\
(\mathrm{V})\end{array}$ & $\begin{array}{c}\mathrm{U}_{\mathrm{B}} \\
(\mathrm{V})\end{array}$ & $\begin{array}{c}\mathrm{U}_{\mathrm{D}} \\
(\mathrm{V})\end{array}$ & $\begin{array}{c}\mathrm{U}_{\mathrm{C}} \\
(\mathrm{V})\end{array}$ & $\begin{array}{c}\mathrm{U}_{\mathrm{E}} \\
(\mathrm{V})\end{array}$ \\
\hline 42 & 46.1 & 41.4 & 16.1 & 24.1 & 40.2 \\
50 & 54.8 & 48.2 & 16.2 & 24.2 & 40.4 \\
55 & 60.2 & 52.2 & 16.2 & 24.2 & 40.4 \\
\hline
\end{tabular}

\subsubsection{Experiment of the System}

According to the protection principle of additional DC power supply detection and the designed DC power supply, when the grid voltage is $1140 \mathrm{~V}$, while the ground capacitances are when $0.1 \mu \mathrm{F}$ and $1 \mu \mathrm{F}$ respectively, the corresponding values of UL and leakage resistance RL can be measured as shown in Table 3 and Table 4:

Table 3. Corresponding Values of UI and RI When Ground Capacitance $\mathrm{C}=0.1 \mu \mathrm{F}$

\begin{tabular}{rccc}
\hline$R_{L}$ & $U_{L}(\mathrm{~V})$ & $R_{L}$ & $U_{L}(\mathrm{~V})$ \\
$(\mathrm{K} \Omega)$ & & $(\mathrm{K} \Omega)$ & \\
\hline 0 & 4.8 & 120 & 1.173 \\
20 & 3.18 & 270 & 0.625 \\
40 & 2.37 & 680 & 0.266 \\
80 & 1.573 & 1000 & 0.181 \\
\hline
\end{tabular}

Table. 4 Corresponding Values of UI and RI When Ground Capacitance $\mathrm{C}=0.1 \mu \mathrm{F}$

\begin{tabular}{cccc}
\hline$R_{L}(\mathrm{~K} \Omega)$ & $U_{L}(\mathrm{~V})$ & $R_{L}(\mathrm{~K} \Omega)$ & $U_{L}(\mathrm{~V})$ \\
\hline 0 & 4.81 & 120 & 1.174 \\
20 & 3.17 & 270 & 0.624 \\
40 & 2.37 & 680 & 0.265 \\
80 & 1.574 & 1000 & 0.182 \\
\hline
\end{tabular}

It can be seen from the test data of above table: 
(1) The ground capacitance of the grid has no effect on additional DC detection, which means that it does not affect the action resistance of leakage blocking.

(2) The level of UL can be used to indirectly reflect the insulation level of the grid.

Leakage blocking threshold voltage can be drawn $U_{L}=2.37 \mathrm{~V}$ from leakage blocking resistance $R_{L}=40 K \Omega$. When the resistance of slide-wire rheostat that is artificially added is decreased slowly, the system simulates the occurrence of leakage fault, and the system displays the "leakage fault in X circuit". The test results are shown in Table 5.

Table 5. Test Values of Leakage Blocking Under Different Voltages

\begin{tabular}{|c|c|c|c|c|}
\hline \multirow{3}{*}{$\begin{array}{l}\text { Voltage } \\
\text { (V) }\end{array}$} & \multicolumn{2}{|c|}{ Resistance of single-phase blocking } & \multicolumn{2}{|c|}{ Resistance of single-phase unlocking } \\
\hline & $\begin{array}{c}\text { Required value } \\
(\mathrm{K} \Omega)\end{array}$ & $\begin{array}{l}\text { Test value } \\
(\mathrm{K} \Omega)\end{array}$ & $\begin{array}{r}\text { Required } \\
\text { value }(\mathrm{K} \Omega)\end{array}$ & $\begin{array}{l}\text { Test value } \\
(\mathrm{K} \Omega)\end{array}$ \\
\hline & $\leq 40+8$ & 41 & $<60$ & 41.6 \\
\hline $0.75 \mathrm{U}_{\mathrm{N}}$ & $\leq 40+8$ & 41.6 & $<60$ & 42 \\
\hline $\begin{array}{c}\mathrm{U}_{\mathrm{N}} \\
1.15 \mathrm{U}_{\mathrm{N}}\end{array}$ & $\leq 40+8$ & 41.9 & $<60$ & 42.5 \\
\hline
\end{tabular}

The data in the table are average values of 10 tests. It can be seen from test values that blocking resistance is consistent with the standard requirements.

\subsection{Zero-Sequence Power Directional Protection}

Zero-sequence power directional protection is a selective leakage protection method, by which whether there is asymmetric leakage in the grid can be determined, and the most important is that it can determine the leakage branch, with selecting function of leakage.

The principle of protection with zero-sequence power directional protection is shown in Figure 6. When leakage fault occurs in one branch of underground low-voltage grid, the first step is to sample zero-sequence current and zero-sequence voltage signals by the sampling circuit in the grid, and then the signals are amplified and shaped through the conditioning circuit, which are collected by AD. Fourier algorithm is used to determine leakage branch, and leakage fault is cut off to realize selective leakage protection.

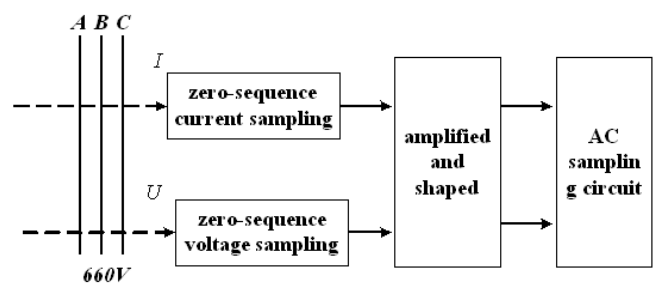

\section{Figure 6. Principle of Zero-Sequence Power Directional Protection}

Assuming the phase angles of zero-sequence current $I_{0}$ and zero-sequence voltage $U_{0}$ are $\varphi_{i}$ and $\varphi_{u}$ respectively, the values of them are worked out according to Fourier algorithm:

$$
\begin{array}{lll}
\varphi_{i}=\arctan \frac{I_{0 I}}{I_{0 R}} & \text { (Equation 6) } \\
\varphi_{u}=\arctan \frac{I_{0 I}}{U_{0 R}} & \text { (Equation 7) }
\end{array}
$$

Making $\varphi=\varphi_{u}-\varphi_{i}$, then we have

$$
\sin \varphi=\frac{I_{0 R} U_{0 I}-I_{0 I} U_{0 R}}{\sqrt{\left(I_{0 I}^{2}+I_{0 R}^{2}\right)\left(U_{0 I}^{2}+U_{0 R}^{2}\right)}} \quad \text { (Equation 8) }
$$


$U_{O I}, U_{O R}, I_{O I}$ and $I_{O R}$ are the real and imaginary parts of $U_{0}$ and $I_{0}$ respectively.

The phase of zero-sequence current $I_{0}$ is shifted forward by $90^{\circ}$. Fundamental wave zero-sequence voltage $U_{0}$ is taken as base and $I_{0}$ is projected to the plane parallel to $U_{0}$, to form the following criteria equation for the leakage selection of leakage protection:

$$
P_{r}=U_{01} I_{01} \cos \left(\varphi+90^{\circ}\right) \quad \text { (Equation 9) }
$$

$U_{01}$ and $I_{01}$ are module values of zero-sequence voltage $U_{0}$ and zero-sequence current sampling $I_{0} ; U_{01}=\sqrt{\left(U_{0 I}^{2}+U_{0 R}^{2}\right)}$, and $I_{01}=\sqrt{\left(I_{0 I}^{2}+I_{0 R}^{2}\right)}$, which can be further simplified as:

$$
P_{r}=U_{0 R} I_{0 I}-U_{0 I} I_{0 R} \quad \text { (Equation 10) }
$$

From the analysis of the zero-sequence current in single-phase ground of low-voltage power supply system, it can be known $P_{r}=U_{0} \Sigma I_{C}$ in the fault circuit, while in a non-fault circuit, $P_{r}=U_{0} \Sigma I_{C 2} ; P_{r}>0$ corresponding to the fault branch, and $P_{r}<0$ for non-fault branch; moreover, the value of $P_{r}$ in the fault branch is much greater than that in non-fault branch, from which the fault branch can be interpreted accurately and reliably.

The advantage of this selecting method is to free of the requirement in the current transformer accuracy from zero-sequence current as the obtained by calculating the zerosequence power is the product of the obtained voltage and zero-sequence current of each branch after moving back of the zero-sequence voltage in the bus by $90^{\circ}$, thus improving judgment accuracy.

\section{Conclusion}

In this paper, the design of leakage protection system of mine low-voltage grid is proposed based on the combination of ZigBee protocol the framework of Internet of Things, and the perception layer of Internet of Things is constructed by ZigBee technology to collect and transmit the related data. The low-voltage leakage fault principle of the mine and the variation law of zero-sequence current and zero-sequence voltage mine are analyzed in this paper; on this basis, a selective leakage protection scheme based on the additional DC power detection and zero-sequence power direction is proposed, in which additional DC power principle is applied to the main feeder switch, the protection principle of zero-sequence power direction is applied to the branch feeder switch, and the branch leakage is interpreted by Fourier algorithm. Through experimental verification, the performance indicators of leakage protection system are consistent with standard requirements, with the small error, which can meet the requirements of the mine grid leakage protection. Internet of Things can realize ZigBee networking and transmit the leakage information of the grid the host computer through ZigBee network.

\section{References}

[1] D. A. Visan, M. Jurian and I. B. Cioc, "Wireless measurement system based on ZigBee transmission technology Electronics Technology (ISSE)”, International Spring Seminar, vol. 33, (2010) , pp. 464-467.

[2] S. Farahani, "ZigBee Wireless Networks and Transceivers", the United States of America, Newness Publications, (2008).

[3] C. Kai, Z. Yi and H. Jianhua, "A localization scheme for underwater wireless sensor networks", International Journal of Advanced Science and Technology, vol. 4, (2009), pp. 9-16.

[4] M. Santiago, L. Francisco, F. Patrida, B. Alfonso and B. Juan, "Ranking of TOA measurements based on the estimate of the NLOS propagation contribution in a wireless location system", Wireless Personal Communications, vol. 3, (2010), pp. 35-52.

[5] M. E. Baran and I. M. EI-Markaby, "Fault analysis on distribution feeders with distributed generations", IEEE Transactions on Power System, vol. 20, no. 4, (2005), pp. 1757-1764.

[6] X. Y. Dou and C. M. Li, "Simulation on faulty line selection of small current grounding system Based on wavelet", Power and energy engineering conference, Chengdu, (2010), pp. 1-4. 
[7] V. M. Prodano and T. C. Green, "High-quality power generation through distributed control of a power park micro grid”, IEEE Transactions on Industrial Electronics, vol. 53, no. 5, (2006), pp. 1471-1482.

\section{Author}

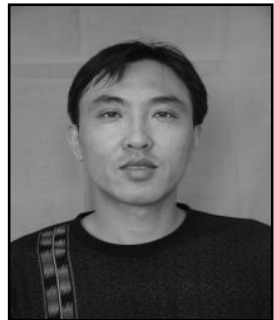

Liu Zhongfu, born in 1973, received the Master degree in Power Electronics and Motor Drives from Taiyuan University of Technology in 2003. Now he is a lecturer at College of Electric Information Engineering, Dalian Nationalities University, China. His papers have been published in some well-known international Journals. His main interests include Embedded System, Technical application of Internet of Things. 
International Journal of Smart Home

Vol. 9, No. 7 (2015) 